\title{
BMJ Open Systematic evidence review of rates and burden of harm of intravenous admixture drug preparation errors in healthcare settings
}

\author{
Nancy Hedlund, ${ }^{1}$ Idal Beer, ${ }^{2}$ Torsten Hoppe-Tichy, ${ }^{3}$ Patricia Trbovich ${ }^{4,5}$
}

To cite: Hedlund N, Beer I, Hoppe-Tichy T, et al.

Systematic evidence review of rates and burden of harm of intravenous admixture drug preparation errors in healthcare settings. BMJ Open 2017;7:e015912. doi:10.1136/ bmjopen-2017-015912

- Prepublication history and additional material for this paper are available online. To view these files, please visit the journal online (http://dx.doi org/10.1136/bmjopen-2017015912).

Received 11 January 2017 Revised 24 July 2017 Accepted 26 July 2017

\section{CrossMark}

${ }^{1}$ Baxter Healthcare Corporation, Global Health Economics and Outcomes Research, Deerfield, Illinois, USA

${ }^{2}$ Medical Affairs, Baxter Healthcare Corporation, Deerfield, Illinois, USA

${ }^{3}$ Pharmacy Department and Cooperation Unit Clinical Pharmacy, University Hospital of Heidelberg, Heidelberg, Germany

${ }^{4}$ Institute of Health Policy, Medicine and Evaluation, University of Toronto, Toronto, Ontario, Canada

${ }^{5}$ North York General Hospital, Toronto, Ontario, Canada

Correspondence to

Dr Idal Beer;

idal_beer@hotmail.com

\section{ABSTRACT}

Objective To examine published evidence on intravenous admixture preparation errors (IAPEs) in healthcare settings. Methods Searches were conducted in three electronic databases (January 2005 to April 2017). Publications reporting rates of IAPEs and error types were reviewed and categorised into the following groups: component errors, dose/calculation errors, aseptic technique errors and composite errors. The methodological rigour of each study was assessed using the Hawker method.

Results 0 the 34 articles that met inclusion criteria, 28 reported the site of IAPEs: central pharmacies $(n=8)$, nursing wards $(n=14)$, both settings $(n=4)$ and other sites $(n=3)$. Using the Hawker criteria, $14 \%$ of the articles were of good quality, $74 \%$ were of fair quality and $12 \%$ were of poor quality. Error types and reported rates varied substantially, including wrong drug ( 0\% to 4.7\%), wrong diluent solution ( $0 \%$ to $49.0 \%)$, wrong label $(0 \%$ to $99.0 \%)$, wrong dose $(0 \%$ to $32.6 \%)$, wrong concentration $(0.3 \%$ to $88.6 \%)$, wrong diluent volume $(0.06 \%$ to $49.0 \%)$ and inadequate aseptic technique (0\% to $92.7 \%) \%$ ). Four studies directly compared incidence by preparation site and/or method, finding error incidence to be lower for doses prepared within a central pharmacy versus the nursing ward and lower for automated preparation versus manual preparation. Although eight studies (24\%) reported $\geq 1$ errors with the potential to cause patient harm, no study directly linked IAPE occurrences to specific adverse patient outcomes.

Conclusions The available data suggest a need to continue to optimise the intravenous preparation process, focus on improving preparation workflow, design and implement preventive strategies, train staff on optimal admixture protocols and implement standardisation. Future research should focus on the development of consistent error subtype definitions, standardised reporting methodology and reliable, reproducible methods to track and link risk factors with the burden of harm associated with these errors.

\section{INTRODUCTION}

Errors in medication preparation and administration can lead to patient harm. ${ }^{1-3}$ For example, many preventable adverse events with respect to medication have been linked to errors in dosing (eg, patients receiving

\section{Strengths and limitations of this study}

- To the authors' knowledge, this is the first systematic review conducted that attempts to categorise intravenous admixture preparation errors (IAPEs) according to both the characteristics of the error and the location and method of intravenous preparation.

- Although IAPE is a safety concern, its frequency, subtypes and associated burden of harm are not well understood; thus, the current review presented a thoughtful and valid framework to assess IAPEs within their procedural context.

- This review attempted to include all articles published in English between January 2005 and April 2017 that reported on IAPEs in which healthcare professionals prepared $\geq 1$ dose of intravenous administered therapy.

- This review is limited by the number of studies identified that reported data on the frequency and/or burden of harm of IAPEs.

higher or lower amounts of medication than intended). ${ }^{24}$ The medication use cycle for an intravenous medication involves multiple steps prior to administration, including prescribing and transcription (paper-based orders), in addition to a number of admixture preparation and labelling steps (figure 1).

An intravenous admixture preparation error (IAPE) can be considered as any deviation from the specifications involved in the admixture preparation and labelling process. An IAPE is a form of medication error-in other words, a preventable adverse event resulting from inappropriate medication preparation, administration or use that can lead to patient harm, including death, while the medication is in the control of the healthcare professional, patient or consumer. ${ }^{56}$

IAPEs can be introduced at multiple points during admixture preparation and labelling. These steps can occur on site at a nursing ward or in a central or satellite pharmacy. Intravenous medication doses are typically 


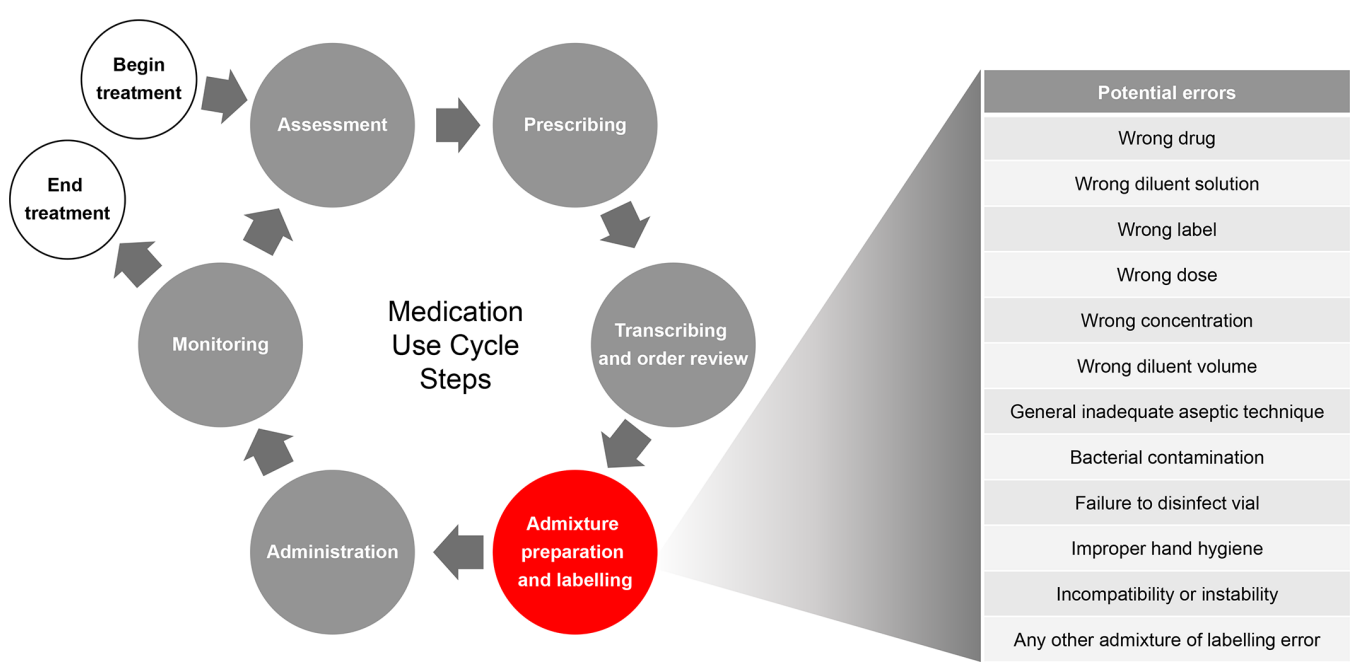

prepared (1) manually by nurses, either at the bedside or in a ward-based preparation room, (2) manually by pharmacists and pharmacy technicians in a central or satellite clean room under a laminar-airflow hood or (3) through the use of pharmacy automation technology, which can be partially or fully automated and may be located in clean rooms or clean compartments within the machine. US data suggest increasing use of automated technologies aimed at reducing IAPEs, for technologies ranging from robotic chemotherapy compounding devices $(0.3 \%$ of hospitals) to barcode verification (20\% of hospitals), with higher levels of adoption predominantly within larger hospitals. ${ }^{7}$ Delivery of the correct dose of an intravenous admixture to a patient depends on the careful control of many factors, such as the calculation of a patient-specific dose (eg, based on body weight or organ function), oversight of procedures used for admixture preparation and labelling practices. ${ }^{4}$ While research suggests that the highest medication-error rates can be attributed to the prescribing and administration phases of the medication use cycle, ${ }^{9-11}$ studies focused on medication preparation practices suggest that the intravenous admixture preparation and labelling phase pose a significant potential for errors. ${ }^{912-15}$ It is unknown what proportion of IAPEs are unreported.

In addition to measuring the incidence of IAPEs, it is also important to understand their impact in terms of burden of harm. Two examples of existing frameworks for categorising patient harm resulting from medication errors are The Institute for Safe Medication Practices (ISMP) high-alert medication lists and The National Coordinating Council for Medication Error Reporting and Prevention (NCC MERP) Medication Error Index. ISMP publishes information and educational resources for healthcare providers on preventing medication errors and tracks voluntary medication errors reports. Based on these voluntary error reports, ISMP maintains lists of high-alert medications in outpatient and inpatient settings that have the potential for increased risk of patient harm if used in error. ${ }^{16}$ The NCC MERP
Medication Error Index groups medication errors into nine possible categories, ranging from non-errors (situations in which errors may occur) to errors resulting in patient death. ${ }^{17}$ These categories also include near-miss (near-hit) situations in which an error occurred but did not reach the patient or cause harm. ISMP uses the NCC MERP Medication Error Index in its medication error database.

Much of the prior published research focusing on the prescription or administration of intravenous therapies has failed to describe or distinguish between errors that arise as a result of the admixture preparation process versus errors associated with incorrect prescribing or administration. ${ }^{18-21}$ With this systematic review, our objective is to identify the incidence of IAPEs (overall and by subtype) reported across institutional healthcare settings and to understand the frequency of error subtypes and associated burden of patient harm attributable to IAPEs as reported in the published literature.

\section{METHODS}

\section{Identification of literature and data sources}

For the purposes of this review, an IAPE was defined as an error or deviation at any step within the admixture preparation process where the drug container was physically handled or manipulated by a healthcare professional. A broad search strategy was developed to identify all studies (published from January 2005 to April 2017) that mention any type of IAPE in an institutional healthcare setting, which included reports relating to wrong drug, wrong diluent solution, wrong label, wrong dose, wrong concentration, wrong diluent volume and inadequate aseptic technique. Dose omission errors were considered to be errors related to administration rather than preparation and, thus, were not included as a focus in this study. Near-miss and actual errors (those that did reach patients) were both included. The review was structured based on the PICOS (patients, intervention, comparator, outcomes and study design) search strategy (table 1). 


\begin{tabular}{ll}
\hline Table 1 PICOS search strategy \\
$\begin{array}{ll}\text { Patient/Problem } & \text { Incorrect preparation of intravenous admixtures within an institutional healthcare setting (acute or long-term } \\
\text { care) by a licensed healthcare professional (nursing and/or pharmacy staff and/or physician) team member }\end{array}$ \\
$\begin{array}{ll}\text { Intervention } & \text { Preparation of an intravenous admixture } \\
\text { Comparison } & \text { Automated versus manual preparation methods (studies were not required to demonstrate both) } \\
& \text { Central pharmacy versus on-unit (on the nursing ward) preparation location (studies were not required to } \\
& \text { demonstrate both) } \\
& \text { Incorrectly prepared or labelled intravenous admixture, which may or may not have reached a patient: } \\
& \text { Wrong drug or diluent } \\
\text { Outcome } & \text { Wrong dose, concentration or volume } \\
& \text { - Contaminated admixture or failure to follow hygiene or sterility protocols } \\
& \text { Inclusion criteria: Observational studies for which numerator (number of doses impacted or number of } \\
& \text { errors) and denominator (number of eligible doses or opportunities for error) are discernible } \\
& \text { Exclusion criteria: Studies in which isolated contamination volumes are reported but for which total batch } \\
\text { size is unknown fail to qualify for consideration } & \text { Error report logs for which number of errors is known but associated number of prepared doses is not also } \\
\text { fail to qualify }\end{array}$
\end{tabular}

PICOS, patients, intervention, comparator, outcomes and study design.

\section{Systematic review process}

Three electronic databases were searched for relevant literature reporting on IAPE: Ovid MEDLINE, EMBASE and International Pharmaceutical Abstracts. The initial search was conducted on 6 February 2014, with supplementation on 4 September 2015 and 26 April 2017 to include articles published during the interim. Aggregate results include articles published in English between January 2005 and April 2017 that involved studies in human subjects in which a healthcare professional prepared $\geq 1$ doses of intravenous administered therapy (medication, including total parenteral nutrition). This date range was selected to include a sufficiently long period to capture the studies of interest, while remaining relevant to current practice in terms of technology and guidelines. Key search terms and limits used in the systematic review are shown in online supplementary table S1. Screenings for relevant literature citations that appeared in the publications were made during the review process to identify any pertinent, additional publications up to April 2017. For this systematic review, references had to meet the inclusion/exclusion criteria detailed in the next section. Duplicate articles were removed electronically prior to manual review. Titles of the papers and abstracts captured in the electronic search results were screened by two reviewers for relevancy according to prespecified criteria. If the titles did not provide sufficient information for screening, the abstract or full-text articles were then reviewed to discern whether the publication met inclusion criteria. All publications that met entry criteria for the review were obtained as full-text articles and then reassessed by the reviewers against the review criteria. The review process was fully compliant with the 2009
Preferred Reporting Items for Systematic Reviews and Meta-Analyses (PRISMA) guidelines. ${ }^{22}$

\section{Inclusion and exclusion criteria}

Publications reporting on a randomised, controlled trial, prospective cohort study, observational quality audit, descriptive study, quasi-experimental study or quality-improvement study were selected for inclusion. Quasi-experimental studies, quality-improvement studies and descriptive studies were eligible if they included sufficient data on the number of doses prepared. While systematic reviews reporting on these study types were not included, their respective reference lists were reviewed to identify potentially relevant studies. Publications were not limited to a single geographic or physical study location and may have occurred in the hospital or any other institutional or outpatient healthcare setting associated with a hospital.

Publications and studies were included for review if they either reported incidence of IAPE or provided sufficient detail for incidence calculation. These errors included incorrectly dispensed medication as well as near-misses that were caught by the study observer prior to administration. Errors also had to originate with a healthcare professional (eg, nurse or pharmacist). Studies reporting patient or informal caregiver medication errors were not included. To be included, studies were required to report original data on IAPEs, including a denominator, to allow for incidence calculations.

Articles that described only errors in prescribing, transcription, administration and monitoring were not included. In addition to all articles that failed 
to meet the aforementioned inclusion criteria, the following article types were also excluded: conference abstracts, case reports, simulations and survey findings.

\section{Data extraction}

The data extracted from relevant articles for analysis included year of publication, country of origin, study period, patient population, definition of error, intravenous preparation location (eg, central or satellite pharmacy or nursing ward), care setting (eg, critical care, general nursing ward), type of therapy, method of error detection and error incidence. Data were extracted and scored independently by two separate reviewers, with introduction of a third reviewer in the case of scoring discrepancies, with all differences being resolved by consensus. Each review team included $\geq 1$ pharmacist for professional knowledge and understanding of drug preparation. The methodological rigour of each study was critically appraised and scored using the Hawker method. ${ }^{23}$ This appraisal tool is simple and particularly adaptable to literature reviews encompassing varied research methodologies. ${ }^{24}$ It employs nine criteria to evaluate for each study: (1) abstract and title; (2) introduction and aims; (3) method and data; (4) sampling; (5) data analysis; (6) ethics and bias; (7) results; (8) transferability or generalisability and (9) implications and usefulness. For each criterion, studies were scored as: good (score 4), fair (score 3), poor (score 2) or very poor (score 1). A mean score was then calculated for each study across all nine criteria, and the overall quality of each study was likewise scored from good to very poor.

For the purposes of this review, IAPEs were grouped into one of four categories based on the characteristics of the error and the location and method of intravenous preparation. Component errors were defined as all those that result from selecting an incorrect ingredient (ie, wrong drug or wrong diluent solution) or applying an incorrect, incomplete or inaccurate label (ie, wrong label) to the admixture. Dose/calculation errors were defined as those involving the use of an incorrect calculation to determine dose and/or diluent amount or the use of a diluent volume not in accordance with the package insert (ie, wrong dose, wrong concentration and wrong diluent volume). Aseptic technique errors involved a breakdown in the process designed to minimise the potential for antimicrobial contamination (ie, inadequate aseptic technique, bacterial contamination, failure to disinfect phial and improper hand hygiene). The category of composite errors was used to describe IAPE rates reported in aggregate, in which the researchers reported an overall rate that included multiple IAPE subtypes. Composite errors included cases in which $>1$ error or type of error was observed in a single preparation.

This study was registered with the PROSPERO international database of systematic reviews (CRD42014010418) to comply with PRISMA guidelines.
RESULTS

Electronic database searches yielded 2018 English language publications for review. Additional sources (hand searches of publication reference lists) identified another three publications for evaluation. After removing duplicates and screening for inclusion and exclusion criteria, 34 articles were included in the final synthesis (figure 2). ${ }^{325-57}$ Of the 34 articles, five (15\%) were rated good quality, 303438454624 (71\%) were fair quality, ${ }^{325-29} 31-333637$ 39-41 $43444851-57$ and four (12\%) were poor quality 354950 after assessment using the Hawker method. The quality of one study ( $3 \%$ ) could not be fully scored due to a missing data table in the available publication. ${ }^{47}$ Details of the Hawker analysis for each study are shown in online supplementary table S2.

\section{Study characteristics}

A summary of the study characteristics, in terms of the setting and methodology, described in the 34 publications is presented in table 2. Collectively, the publications reported international data, with studies spanning Africa, North America, South America, Europe, the Eastern Mediterranean region and the Western Pacific region. Patient populations varied across studies, with both adults and children represented. Studies were conducted mainly in general inpatient or critical care settings, with several in paediatric or haematology units. The majority of publications (21 (62\%)) assessed errors in $>1$ type of intravenous therapy. Additional individual details for each study are shown in online supplementary table S3.

The most common method of detecting errors was direct observation, used in 17 studies $(50 \%),{ }^{3} 25262831-33 \quad 37394144-48 \quad 5255$ and one study used direct observation and analysis of final intravenous admixture concentration. ${ }^{53}$ Other methods included analysis of final concentration in five studies (15\%), ${ }^{27} 30363851$ bacterial culture in four studies $(12 \%),{ }^{29} 344957$ cross-checking in three studies $(9 \%), 354243$ incident reports in three studies $(9 \%)^{405456}$ and chart review in one study. ${ }^{50}$ In several studies using the direct observation method, nurses or pharmacists preparing the intravenous admixtures consented to participate but were not fully aware of the study aims to avoid influencing their behaviour. ${ }^{18} 3945$ Eight studies (24\%) reported on the accuracy of intravenous preparation before and after an intervention, $353641-43454652$ five studies (15\%) compared intravenous admixture preparation locations or methods, ${ }^{30} 36414249$ and the remaining 21 publications $(62 \%)$ were single-arm studies. ${ }^{3}$ 25-29 31-34 37-40 44474850 55-57

A total of 28 studies reported the intravenous preparation site. Of those studies, 14 publications $(38 \%)$ reported preparation on the nursing ward $^{325262831-34374347485255}$ and $8(24 \%)$ reported use of central pharmacies. 2729353640414454 Three studies $(12 \%)$ compared rates of IAPEs in the nursing ward and a central pharmacy ${ }^{3049}$ and one compared IAPEs in the 


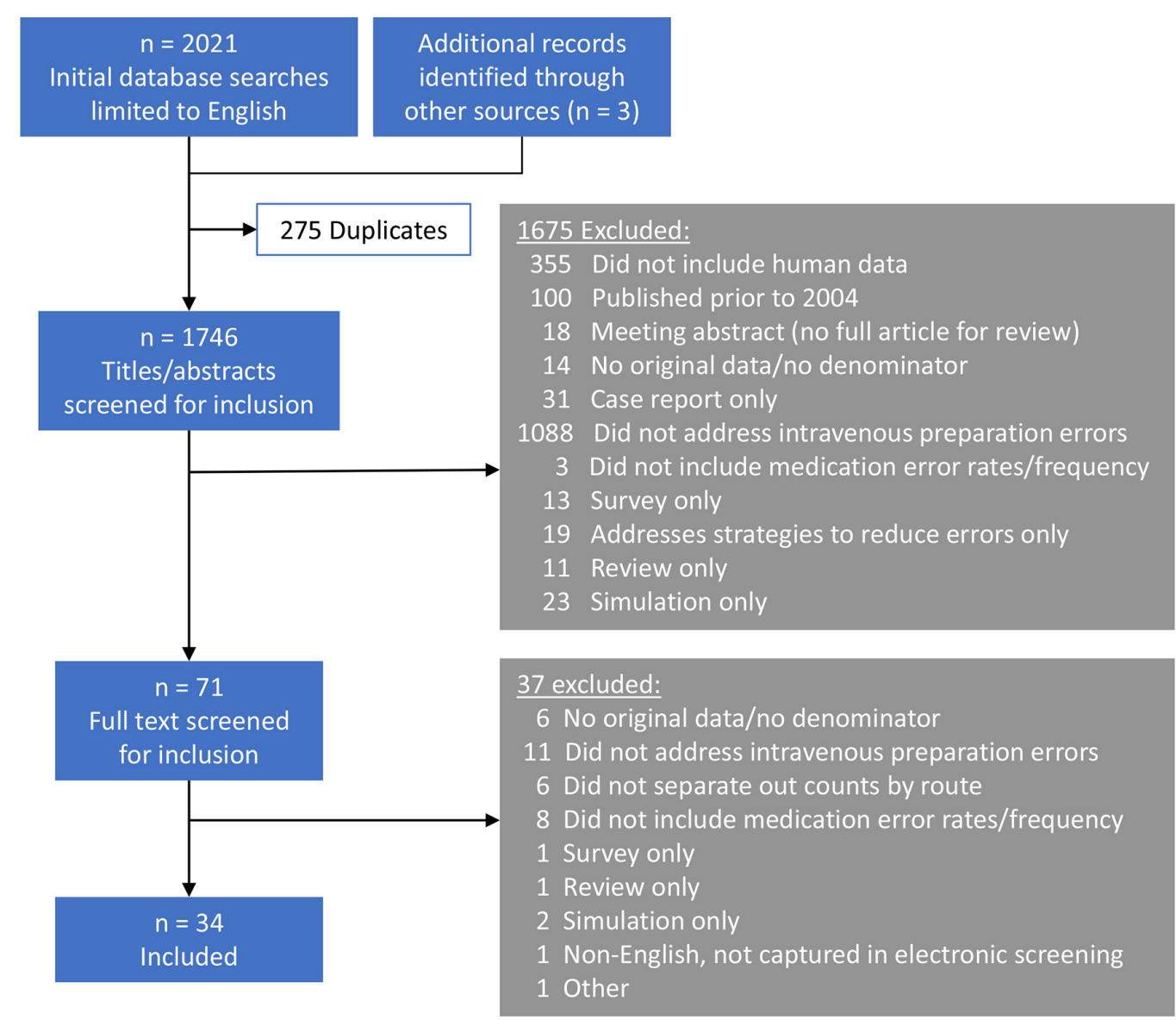

Figure 2 PRISMA study inclusion flow diagram. PRISMA, preferred reporting items for systematic reviews and meta-analysis.

nursing ward and operating theatre.$^{53}$ Last, two studies reported intravenous preparation at offsite pharmacies ${ }^{56}$ and in the obstetric theatre ${ }^{57}$ respectively.

While IAPEs were not consistently linked with individual patient outcomes in the studies surveyed, nearly half of the studies attempted to assess the potential for patient impact in some way. Twelve $(35 \%)$ of the publications included in this review reported on the severity of harm or potential for harm arising from identified IAPEs (see online supplementary table S3), ${ }^{326} 293132$ 39-41 44-46 48 eight $(67 \%)$ of which reported $\geq 1$ errors to result in various degrees of harm $326404144-4648$ and four (33\%) having reported no errors to have resulted in adverse outcomes or to have presented a major patient risk. ${ }^{29} 313239$

Of the 12 studies that reported on burden of harm, three $(25 \%)$ used the NCC MERP medication error index ${ }^{17}$ to score identified errors, ${ }^{31} 3940$ while six studies $(50 \%)$ relied on clinician assessment or an expert panel for the determination of error severity. ${ }^{3}{ }^{26} 4144-46$ Among the six studies that used clinician assessment or an expert panel, two of the study teams (Niemann et $a l^{46}$ and Nguyen et $a t^{45}$ ) assessed errors based on clinical relevance rather than assigning a score based on patient harm or potential for harm. The remaining three studies each took a different approach to estimating patient harm. ${ }^{29} 3248$ Ding and colleagues ${ }^{48}$ were the only authors to record whether the error was associated with a drug found on the ISMP list of high-alert medications. Crill and colleagues ${ }^{29}$ did not have a system for rating error severity but did note that no contamination errors resulted in patient infections. Last, the 2008 study by Fahimi and colleagues ${ }^{32}$ did not describe a specific system for rating error severity, but noted that none of the errors identified resulted in adverse events or major risks to patients. Further detail on how each study assessed patient burden of harm is shown in online supplementary table S4.

\section{Categorisation and incidence of IAPES}

Errors identified in the selected studies were grouped into four broad categories: component errors, dose/calculation errors, aseptic technique errors and composite errors, as detailed in the Methods section. Errors of the same subtype were frequently defined slightly differently among studies; full descriptions of the error subtype definitions are shown in online supplementary table S5. Incidence values for error subtypes are presented in table 3 .

The error subtype of wrong drug selection was infrequent, ${ }^{3} 252831374144455455$ with the highest reported rate of $4.7 \%$ of total doses. ${ }^{31}$ Selection of a wrong diluent solution was reported to have occurred in 9 of 34 publications $(26 \%)$, with results varying across studies ( 0\% to $49.0 \%) .{ }^{32832373944465354}$ Of note, the multicentre, multinational study by Cousins et $a l^{28}$ reported that $1.0 \%-$ $49.0 \%$ of doses administered had been prepared with an 


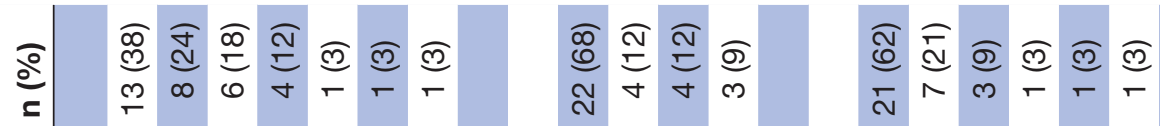

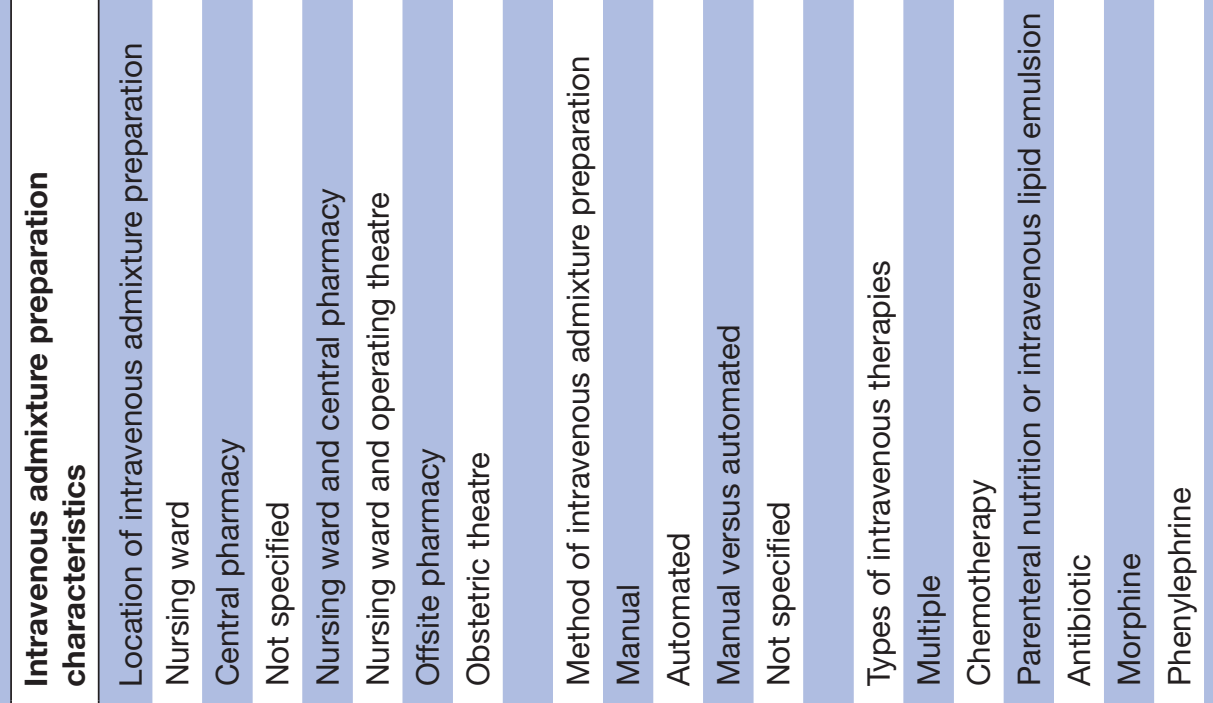

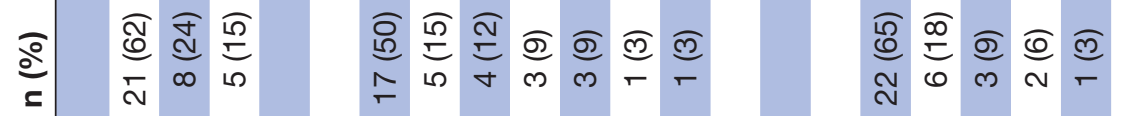

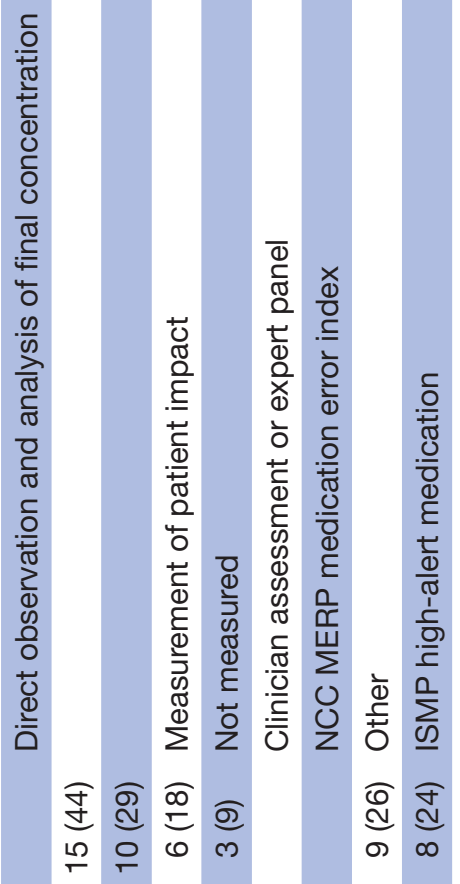

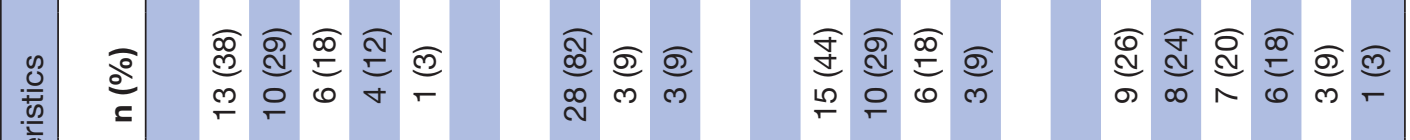
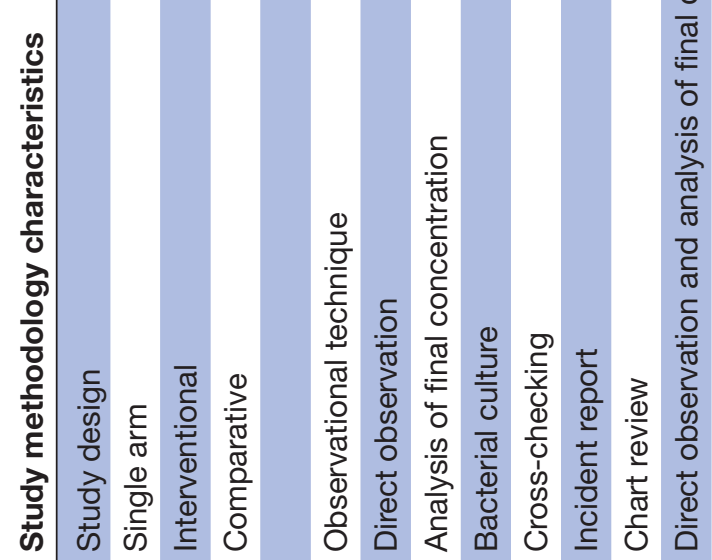

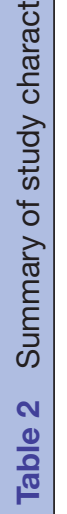
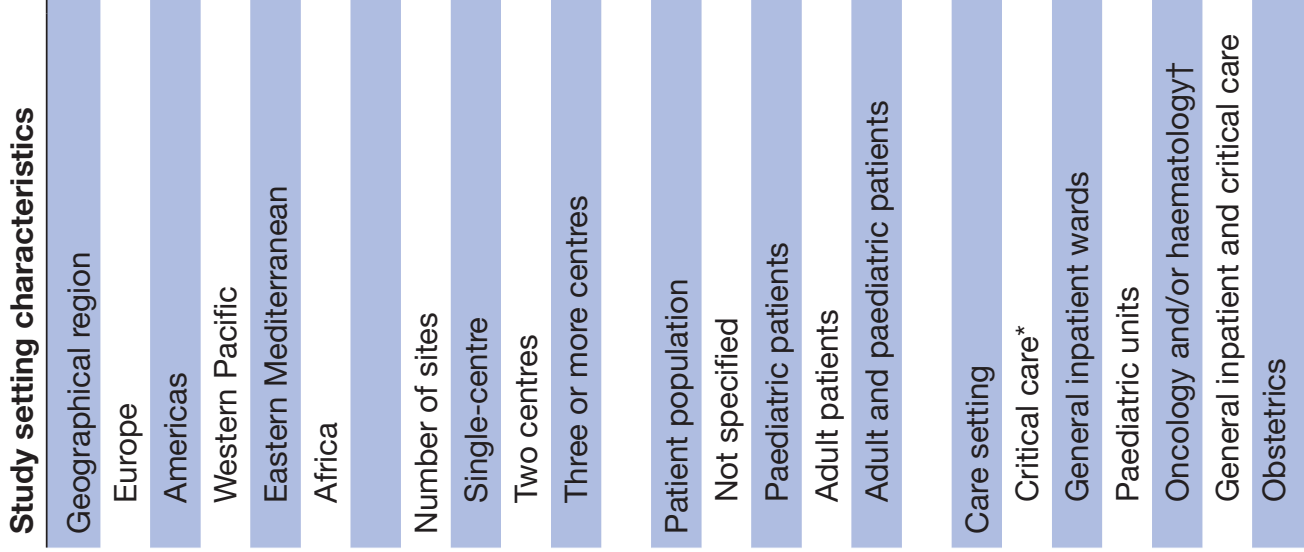

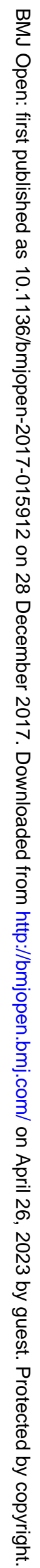




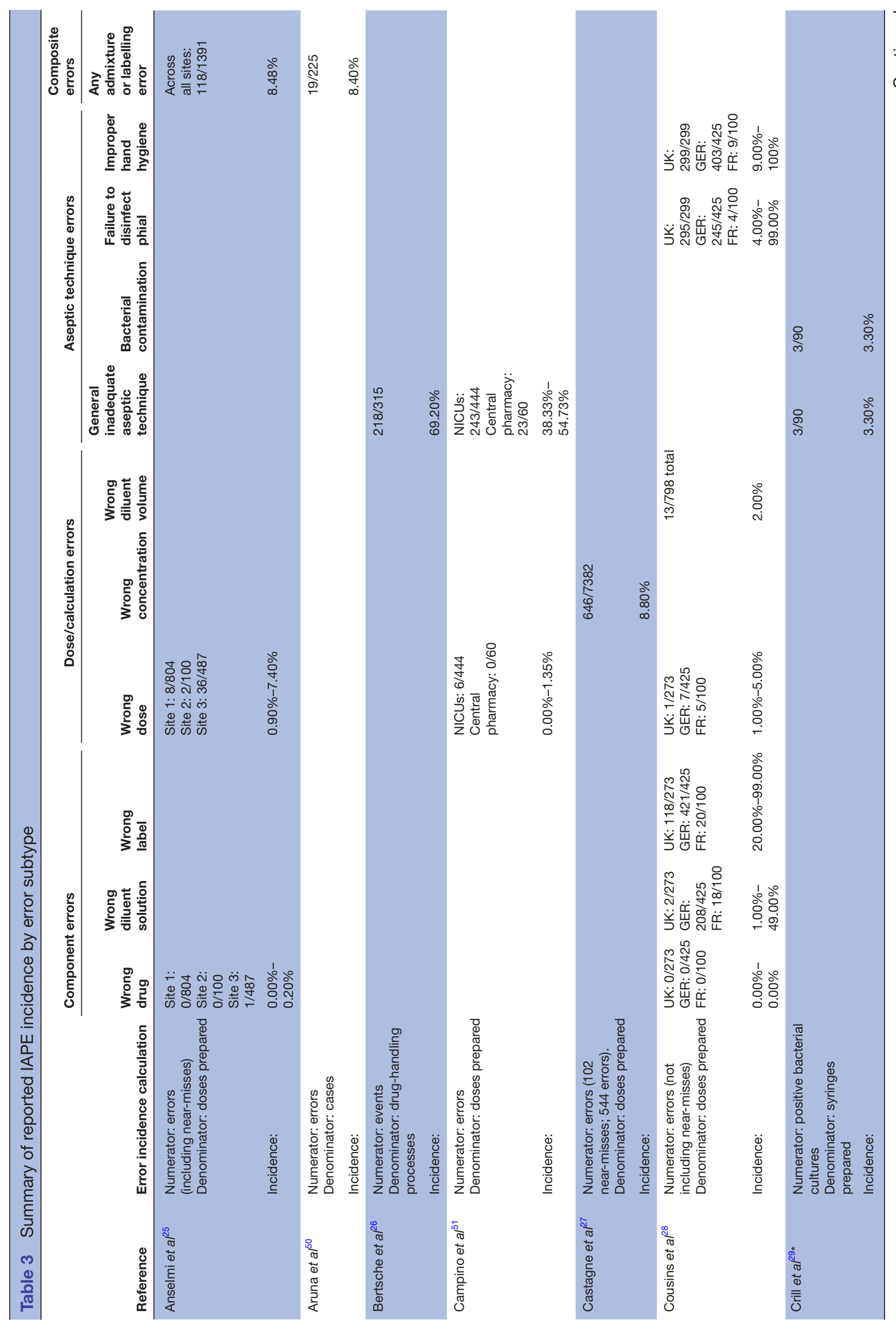




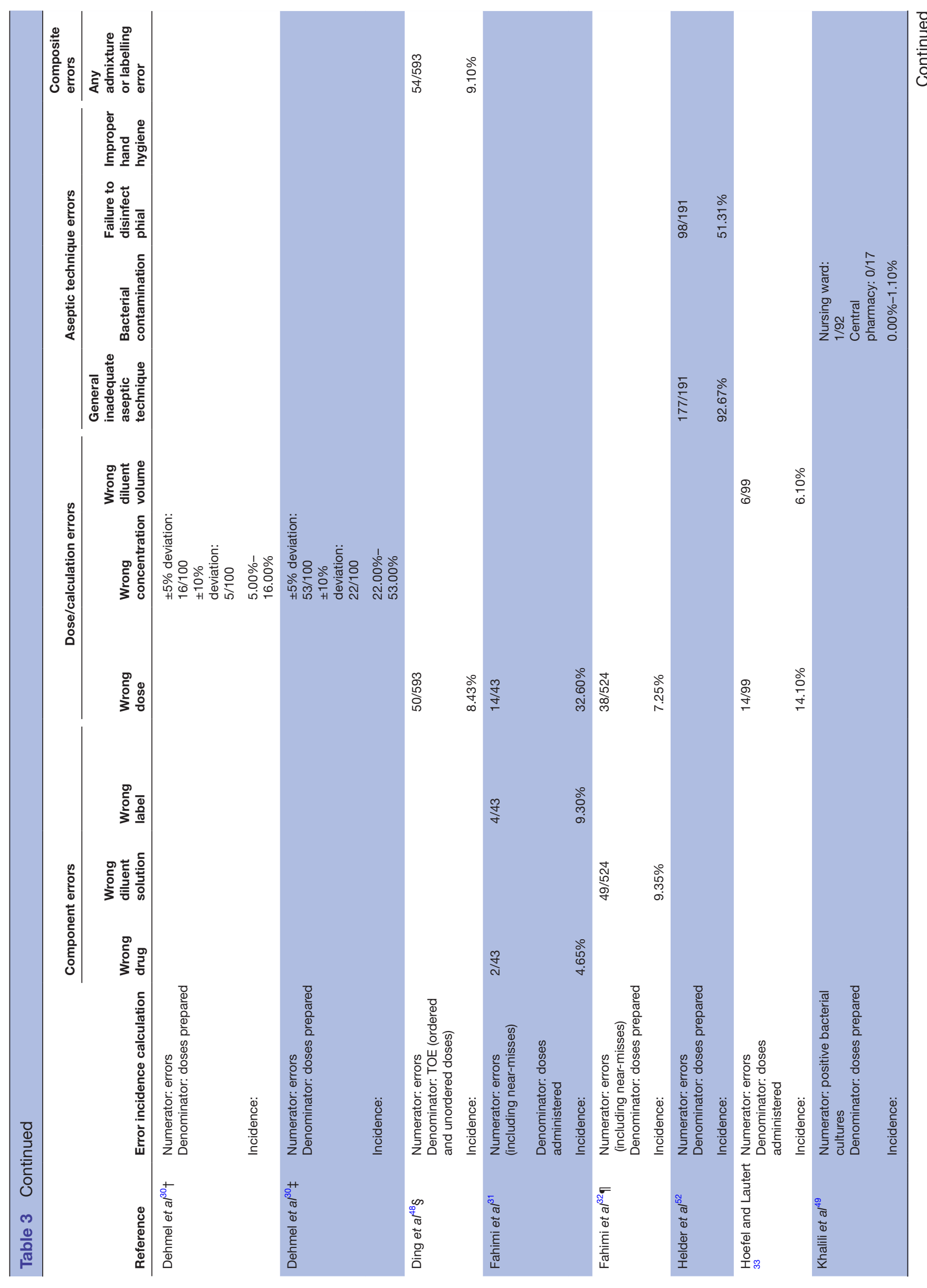

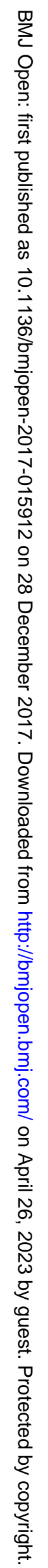




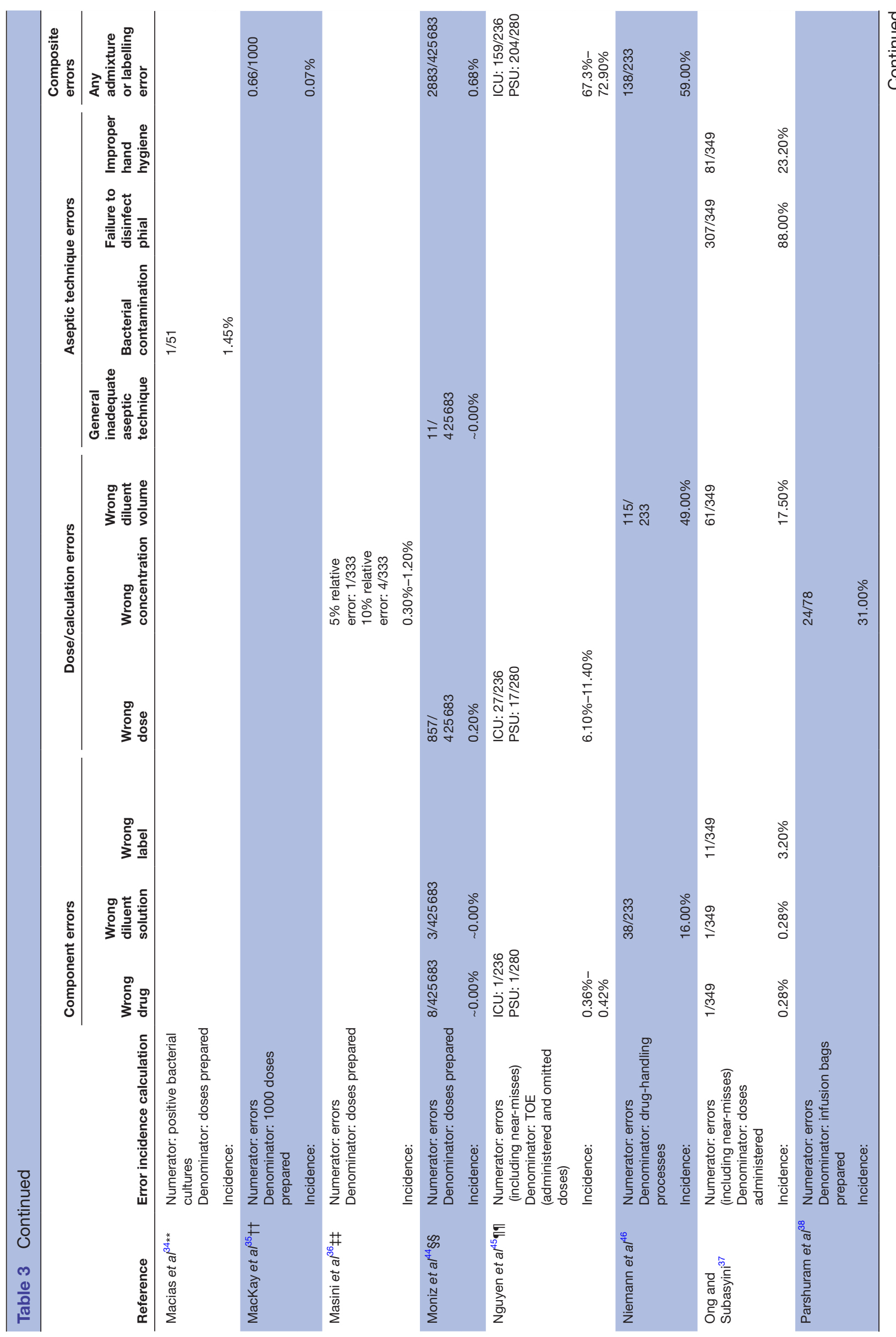

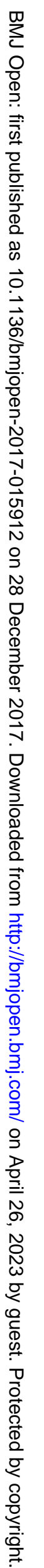



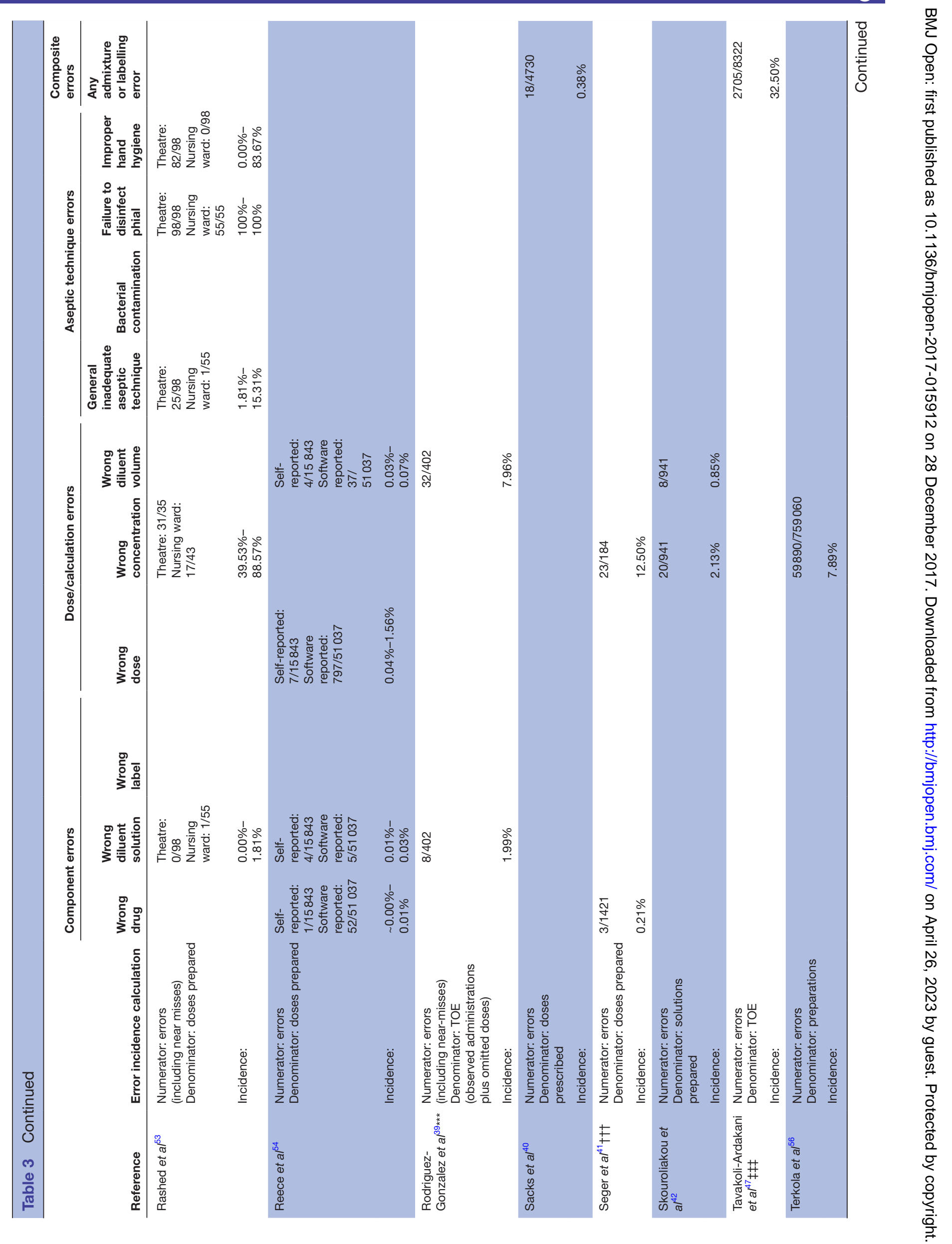

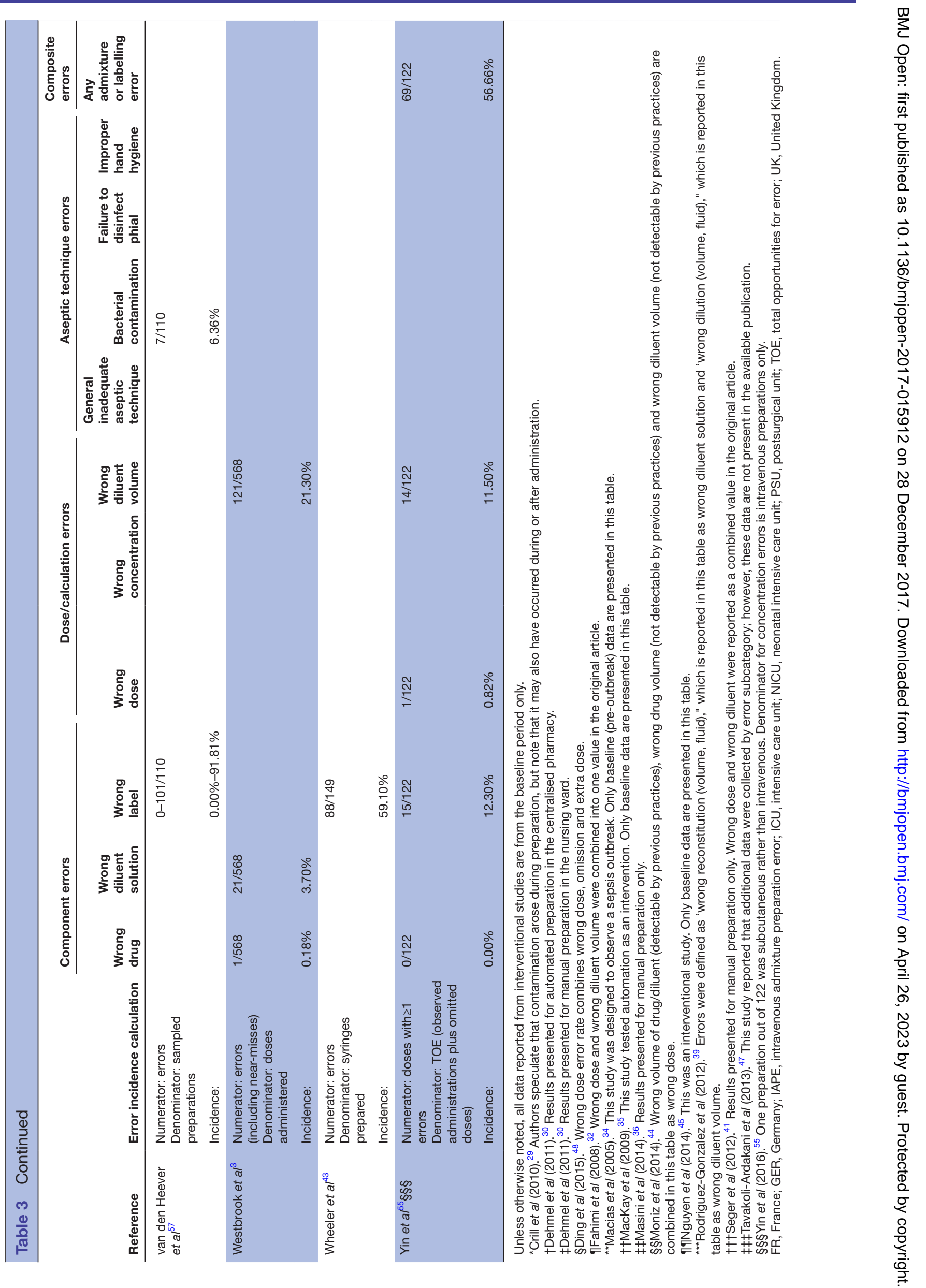
incorrect diluent across all study sites. This range is wider than that of the other included studies $(0 \%-16.0 \%)$. Labelling errors were reported in six publications (18\%), with reported incidence varying substantially, ranging from $0 \%$ to $99.0 \%$ (20.0\% to $99.0 \%$ within the study by Cousins $e t a l^{28}$ and $0 \%$ to $91.8 \%$ in the study by van den Heever et $\left.a \varphi^{57}\right) .^{283137435557}$

Eleven publications (32\%) captured incidence of wrong dose, and while most of these studies reported incidence rates below $10 \%, 25283233444548515455$ one study did report an incidence rate over $32 \% .^{31}$ Wrong drug concentration errors were reported in 10 publications (29\%), with error incidence per total number of intravenous doses prepared ranging from $0.3 \%$ to $88.6 \%$. ${ }^{27} 303638414251535556$ While some studies defined a concentration error based on a threshold of a $5 \%^{303641}$ or $10 \%^{3036385155}$ deviation between the prepared dose and the ideal dose, the study by Castagne et al used a higher threshold of $20 \% .{ }^{27}$

Eight studies (24\%) reported errors pertaining to wrong diluent volume, ${ }^{328333739424654}$ with half explicitly defining this error subgroup as any deviation from manufacturer or accepted institutional guidelines for intravenous preparation. ${ }^{373946}$ The highest-reported error rate $(49.0 \%)$ was identified by Niemann and colleagues, ${ }^{46}$ while the lowest-reported incidence $(0.6 \%)$ was from a study by Reece $e t a l . .^{54}$

Reported challenges with aseptic technique included general aseptic technique deviations, bacterial contamination, failure to disinfect the phial and improper hand hygiene. In studies that reported general inadequate aseptic technique deviations, three studies reported incidence rates below 5\% (range: $0 \%-3.3 \%$ ) $^{29} 4453$; however, the study by Bertsche and colleagues ${ }^{26}$ reported an incidence rate of just under $70 \%$ and findings from Helder et al indicated a $92.7 \%$ non-adherence rate to hygiene protocols. ${ }^{52}$ The variation in incidence rates presented may be the result of differences in error definitions, as Bertsche and colleagues assessed aseptic technique deviations as any procedural deviation from local hygiene guidelines $^{26}$ and a study by Helder et al required all five steps of the hygiene protocol to be followed. ${ }^{52}$ The other studies defined aseptic technique errors either based on bacterial cultures ${ }^{29} 34$ or report of syringes left uncapped during the preparation process. ${ }^{44}$

Bacterial contamination errors were reported in four studies, with all reporting incidence under $7 \%$ (table 3)..$^{29} 344957$ Four additional studies report error incidence for both failure to disinfect the phial ${ }^{28375253}$ and improper hand hygiene. ${ }^{28} 3753$ In particular, the study by Cousins and colleagues ${ }^{28}$ presents a wide range of incidence across aseptic technique subtypes (table 3). The study by Cousins $e t a t^{28}$ presented data from three separate institutions located in France, Germany and UK, with the incidence of aseptic technique errors from the French institution found to be dramatically lower $(4.0 \%$ for phial disinfection and $9.0 \%$ for hand washing). Of note, the authors attribute this difference to the French institution having undergone a recent update to its aseptic preparation methods protocol due to a prior outbreak of Legionnaire's disease within the facility. ${ }^{28}$

Ten $(29 \%)$ studies reported an overall incidence of IAPEs that combined multiple error subtypes. ${ }^{25354044-485055}$ These studies have diverse error definitions and error detection methods; thus, the error incidence ranges widely $(0.07 \%-72.9 \%)$.

\section{DISCUSSION}

This systematic review found that IAPEs are ubiquitous across countries and hospital locations and that the types of errors observed and reported are diverse. Reported error incidence was found to vary widely between settings (central pharmacies or nursing wards) and within these settings across studies. Variability in error detection methods and definitions applied may contribute to the variation in error rates reported across studies.

This review identified studies conducted in Europe, North America, South America, Asia and Africa. While different regions, countries and even individual institutions are likely to have somewhat different standards and practices for intravenous admixture preparation, differences in methods and terms applied for data collection did not seem to vary any greater between countries than within a single country. In theory, variation among institutions within the same country has the potential to be larger than variation among countries, as local practices may be more flexible than nationally adopted standards. ISMP noted in its 2011 Guidelines for the Safe Preparation of Sterile Compounds that intravenous admixture preparation practices are complex, and documentation of errors varies widely across the US. ${ }^{58}$ This highlights an important need for national and international consensuses on defining and identifying IAPEs to fully understand the global patient burden.

Some evidence indicates the effect of location and method of intravenous admixture preparation on the incidence of errors. In particular, error rates appear to be lower when intravenous preparation takes place in central pharmacy settings compared with nursing wards and lower with automated versus manual preparation. Among studies meeting the inclusion criteria for this systematic review, Dehmel and colleagues $^{30}$ and Khalili et at $t^{49}$ directly compared error rates identified from a central pharmacy to those from a nursing ward using consistent IAPE definitions across settings. The study by Dehmel et al reported a markedly higher rate of wrong concentration errors using manual preparation in a nursing ward when compared with automated preparation in a central pharmacy (53\% vs $16 \%$, respectively) ${ }^{30}$ Khalili and colleagues reported a low rate of bacterial contamination $(1.1 \%)$ in admixtures prepared on nursing wards, with no instances of contamination in admixtures prepared in central pharmacies, despite use of manual preparation techniques in each setting. ${ }^{49}$ Caution should be taken in generalising this finding, given the limited sample size of 17 preparations in the central pharmacy and 97 on the nursing ward. ${ }^{49}$ Thus, while it appears that moving intravenous admixture preparation away from the site of care and using 
automated technologies may reduce IAPEs, further empirical studies are required to substantiate this hypothesis.

In the present systematic review of IAPEs, a patchwork of data emerged from the relevant available literature, in part because no single study design or observational technique is ideal for capturing all the aspects of intravenous admixture preparation that could result in an error. The majority of studies relied on direct observation of the intravenous admixture preparation process by a trained observer, while other studies used bacterial culture, measurement of the final admixture concentration, incident reports and cross-checking against a checklist, computed calculation or other benchmarks. However, certain error subtypes naturally lent themselves to a specific observational technique, such as bacterial culture for assessing bacterial contamination, laboratory testing for concentration errors and direct observation for aseptic technique deviations.

The framework used for categorising IAPEs in this review was developed to facilitate the aggregation of data collected across studies. While inconsistency across reported error definitions precluded additional quantitative aggregation, we hope the classification system used herein is informative to researchers designing future studies and may help to facilitate more effective standardisation of error reporting going forward.

Within IAPE subtypes, the method of error calculation varied in some cases, which impacted the ability to generalise results across studies. The majority of studies reported the incidence as errors per doses prescribed, prepared or administered. However, five (15\%) studies reported errors per total opportunities for error 395474855 and two (6\%) studies reported errors per total drug-handling processes. ${ }^{26}{ }^{46}$ While using total opportunities for error or drug-handling processes may be insightful for those wishing to understand and optimise the intravenous medication use cycle from the user perspective, errors per dose may be a more useful measurement for researchers interested in patient impact and outcomes.

Error definitions were also variable within some error subtypes. For instance, thresholds for determining concentration errors ranged from $\pm 5 \%$ variance from the label specification to as high as $\pm 20 \%$ variance. ${ }^{27} 303638414251535556$ Studies reporting IAPE incidence based on a composite of IAPE subtypes were often composed of common elements (eg, wrong drug, wrong concentration), but were sufficiently different that they could not be directly compared. This finding exposes a need for a standardised taxonomy of error subtypes that can be used across a variety of research settings and countries to facilitate meaningful comparisons.

Other factors that may impact error incidence are circumstances, such as either a recent training or sentinel event as described by a study by Cousins et $a l^{28}$ when commenting on proportionally lower aseptic technique deviations observed in the French study site. It was suggested that this finding may be attributed to recent staff training and updated guidelines in the French institution included in the study, prompted by a recent outbreak of Legionnaire's disease at that site. This highlights the impact of staff training as a source of potential regional or institutional error variation and as a means of reducing error rates. Given the short duration between staff training and study implementation, the long-term sustainability of error reduction potentially gained by staff training in the study by Cousins $e t$ al was unclear.

In addition to heterogeneous error incidence results, the articles captured in this systematic review used a variety of approaches to measure the potential burden of patient harm. Several studies used the existing NCC MERP error index ${ }^{17}$ to rate and score errors, and the majority of other studies relied on either local clinician opinion or expert panel. As a result, there is a high degree of variability in terms of how the errors are scored and how potential for patient risk is attributed.

Of the 34 studies included in this review, 12 (35\%) provided estimates or general assessments for potentially attributable patient harm or clinical relevance for IAPEs. ${ }^{3} 262931$ 32 39-41 44-46 48 Effective and standardised traceability measures are required to link a defect in the admixture process that occurs early within the medication use cycle with later negative patient outcomes. Given the separation in time and physical location between admixture preparation and potential patient physical adverse response, it can be challenging to link potential negative patient outcomes to the admixture/compounding process where unrecognised potential errors may exist. ${ }^{12}$ There is a need for robust study designs that allow for the assessment of the association between specific errors incidences and patient outcomes.

Several limitations were present in this systematic review. Our search strategy targeted the broad medical literature, but inclusion of additional databases, such as the Cumulative Index of Nursing and Allied Health Literature, may have added nursing publications relevant to this topic. While the quality of publications was generally fair, only five studies $(15 \%)$ were deemed to be of good quality in terms of methodology and reporting. ${ }^{30} 34384546$ Furthermore, the Hawker method of quality ascertainment is generic and may not be best suited to capturing the unique challenges of this research topic. Drawing comparisons between the studies remains difficult due to substantial variations in error definitions. As a result, meta-analysis of the current IAPE literature was not considered appropriate. Last, in the majority of studies, documentation of error severity and associated burden of harm was not sufficient to allow for a thorough evaluation of the impact on patient care or the consequences for healthcare facilities.

\section{CONCLUSION}

This systematic review is the first to categorise IAPEs according to the characteristics of the error and the location and method of intravenous preparation. It is our hope that future studies may use these categorisations to 
provide a meaningful framework to assess IAPEs within their procedural context. With improved standardisation of IAPE definitions, grouping error subtypes as we have done may facilitate an improved understanding of where errors happen within the intravenous preparation process and devising solutions to help eradicate them. There is a clear potential burden of harm for patients resulting from IAPEs, and thus a need to continue to optimise the intravenous preparation process, focusing on improving preparation workflow, designing and implementing preventive strategies, staff training and implementing process standardisation where possible. Future research should focus on the development of consistent error subtype definitions and a standardised reporting methodology as well as reliable and reproducible methods to track and link risk factors and the burden of harm associated with these errors.

Acknowledgements The authors would like to thank Diane Nitzki-George and Denise Hefley of DNG Consulting for their assistance rendered in this study. Editorial assistance was provided by 0xford PharmaGenesis and was funded by Baxter Healthcare.

Contributors NH: concept/design, data analysis/interpretation, critical revision of article, approval of article. IB: data interpretation, critical revision of article, approval of article. TH-T and PT: concept/design, data interpretation, critical revision of article, approval of article.

Funding This study was sponsored by Baxter Healthcare.

Competing interests $\mathrm{NH}$ is a former employee and stockholder of Baxter Healthcare. IB is a former employee and stockholder of Baxter Healthcare. TH-T has no relevant competing interests to disclose. PT is currently under contract to perform other work for Baxter Healthcare that is unrelated to the current manuscript.

Provenance and peer review Not commissioned; externally peer reviewed.

Data sharing statement As the research presented is a systematic literature review of published data, no additional unpublished data are available.

Author note NH and IB are former employees of Baxter Healthcare Corporation.

Open Access This is an Open Access article distributed in accordance with the Creative Commons Attribution Non Commercial (CC BY-NC 4.0) license, which permits others to distribute, remix, adapt, build upon this work non-commercially, and license their derivative works on different terms, provided the original work is properly cited and the use is non-commercial. See: http://creativecommons.org/ licenses/by-nc/4.0/

(c) Article author(s) (or their employer(s) unless otherwise stated in the text of the article) 2017. All rights reserved. No commercial use is permitted unless otherwise expressly granted.

\section{REFERENCES}

1. Leape LL, Brennan TA, Laird N, et al. The nature of adverse events in hospitalized patients. Results of the Harvard Medical Practice Study II. N Engl J Med 1991;324:377-84.

2. Phillips J, Beam S, Brinker A, et al. Retrospective analysis of mortalities associated with medication errors. Am J Health Syst Pharm 2001;58:1835-41.

3. Westbrook Jl, Rob Ml, Woods A, et al. Errors in the administration of intravenous medications in hospital and the role of correct procedures and nurse experience. BMJ Qual Saf 2011;20:1027-34.

4. ISMP Canada. Managing overfill during preparation and delivery of intravenous medications. ISMP Canada Safety Bulletin 2013;69:16-9. https://www.ismp-canada.org/download/safety Bulletins/2013/ISMPCSB2013-07_ManagingOverfillIntravenousM edications.pdf

5. Flynn EA, Pearson RE, Barker KN. Observational study of accuracy in compounding i.v. admixtures at five hospitals. Am J Health Syst Pharm 1997;54:904-12.
6. The National Coordinating Council for Medication Error Reporting and Prevention. The council: moving into the second decade "developing recommendations and offering tools.". 2010 http://www. nccmerp.org/sites/default/files/fifteen_year_report.pdf (accessed 13 Jun 2017).

7. Pedersen CA, Schneider PJ, Scheckelhoff DJ. ASHP national survey of pharmacy practice in hospital settings: Dispensing and administration-2014. Am J Health Syst Pharm 2015;72:1119-37.

8. Speth SL, Fields DB, Schlemmer CB, et al. Optimizing i.v. workflow. Am J Health Syst Pharm 2013;70:2076-80.

9. Leape LL Bates DW, Cullen DJ, et al. Systems analysis of adverse drug events. JAMA 1995;274:35-43.

10. Bates DW, Boyle DL, Vander Vliet MB, et al. Relationship between medication errors and adverse drug events. $J$ Gen Intern Med 1995;10:199-205.

11. Walsh KE, Dodd KS, Seetharaman K, et al. Medication errors among adults and children with cancer in the outpatient setting. J Clin Oncol 2009;27:891-6.

12. White R, Cassano-Piché A, Fields A, et al. Intravenous chemotherapy preparation errors: patient safety risks identified in a pan-Canadian exploratory study. J Oncol Pharm Pract 2014;20:40-6.

13. Walsh KE, Kaushal R, Chessare JB. How to avoid paediatric medication errors: a user's guide to the literature. Arch Dis Child 2005;90:698-702.

14. Bertoglio S, Rezzo R, Merlo FD, et al. Pre-filled normal saline syringes to reduce totally implantable venous access deviceassociated bloodstream infection: a single institution pilot study. $J$ Hosp Infect 2013;84:85-8.

15. Macias AE, Huertas M, de Leon SP, et al. Contamination of intravenous fluids: a continuing cause of hospital bacteremia. $A m \mathrm{~J}$ Infect Control 2010;38:217-21.

16. ISMP. ISMP list of high-alert medications in acute care settings. 2014 https://www.ismp.org/tools/institutionalhighAlert.asp (accessed 13 Jun 2017).

17. National Coordinating Council for Medication Error Reporting and Prevention. NCC MERP index for categorizing medication errors. 2001 http://www.nccmerp.org/sites/default/files/indexBW2001-0612.pdf (accessed 13 Jun 2017).

18. Cousins $D H$, Gerrett $D$, Warner B. A review of medication incidents reported to the National Reporting and Learning System in England and Wales over 6 years (2005-2010). Br J Clin Pharmacol 2012;74:597-604.

19. Abbasinazari M, Zareh-Toranposhti S, Hassani A, et al. The effect of information provision on reduction of errors in intravenous drug preparation and administration by nurses in ICU and surgical wards. Acta Med Iran 2012;50:771-7.

20. Chua SS, Tea MH, Rahman MH. An observational study of drug administration errors in a Malaysian hospital (study of drug administration errors). J Clin Pharm Ther 2009;34:215-23.

21. Prot S, Fontan JE, Alberti C, et al. Drug administration errors and their determinants in pediatric in-patients. Int J Qual Health Care 2005;17:381-9.

22. Moher D, Liberati A, Tetzlaff $J$, et al. Preferred reporting items for systematic reviews and meta-analyses: the PRISMA statement. PLoS Med 2009;6:e1000097.

23. Hawker S, Payne S, Kerr C, et al. Appraising the evidence: reviewing disparate data systematically. Qual Health Res 2002;12:1284-99.

24. Green J, Jester R, McKinley R, et al. The impact of chronic venous leg ulcers: a systematic review. J Wound Care 2014;23:601-12.

25. Anselmi ML, Peduzzi M, Dos Santos CB. Errors in the administration of intravenous medication in Brazilian hospitals. J Clin Nurs 2007;16:1839-47.

26. Bertsche T, Niemann D, Mayer Y, et al. Prioritising the prevention of medication handling errors. Pharm World Sci 2008;30:907-15.

27. Castagne V, Habert H, Abbara C, et al. Cytotoxics compounded sterile preparation control by HPLC during a 16-month assessment in a French university hospital: importance of the mixing bags step. $J$ Oncol Pharm Pract 2011;17:191-6.

28. Cousins DH, Sabatier B, Begue D, et al. Medication errors in intravenous drug preparation and administration: a multicentre audit in the UK, Germany and France. Qual Saf Health Care 2005; $14: 190-5$

29. Crill CM, Hak EB, Robinson LA, et al. Evaluation of microbial contamination associated with different preparation methods for neonatal intravenous fat emulsion infusion. Am J Health Syst Pharm 2010;67:914-8.

30. Dehmel C, Braune SA, Kreymann G, et al. Do centrally pre-prepared solutions achieve more reliable drug concentrations than solutions prepared on the ward? Intensive Care Med 2011;37:1311-6.

31. Fahimi F, Sistanizad M, Abrishami R, et al. An observational study of errors related to the preparation and administration of medications 
given by infusion devices in a teaching hospital. Iran $J$ Pharm Res 2007;6:295-9.

32. Fahimi F, Ariapanah P, Faizi M, et al. Errors in preparation and administration of intravenous medications in the intensive care unit of a teaching hospital: an observational study. Aust Crit Care 2008;21:110-6.

33. Hoefel HH, Lautert L. Errors committed by nursing technicians and assistants in administering antibiotics. Am J Infect Control 2006;34:437-42.

34. Macias AE, Munoz JM, Galvan A, et al. Nosocomial bacteremia in neonates related to poor standards of care. Pediatr Infect Dis $J$ 2005;24:713-6.

35. MacKay MW, Cash J, Farr F, et al. Improving pediatric outcomes through intravenous and oral medication standardization. $J$ Pediatr Pharmacol Ther 2009;14:226-35.

36. Masini C, Nanni O, Antaridi S, et al. Automated preparation of chemotherapy: quality improvement and economic sustainability. Am $J$ Health Syst Pharm 2014;71:579-85.

37. Ong WM, Subasyini S. Medication errors in intravenous drug preparation and administration. Med J Malaysia 2013;68:52-7.

38. Parshuram CS, Dupuis LL, To T, et al. Occurrence and impact of unanticipated variation in intravenous methotrexate dosing. Ann Pharmacother 2006;40:805-11.

39. Rodriguez-Gonzalez CG, Herranz-Alonso A, Martin-Barbero ML, et al. Prevalence of medication administration errors in two medical units with automated prescription and dispensing. J Am Med Inform Assoc 2012;19:72-8.

40. Sacks GS, Rough S, Kudsk KA. Frequency and severity of harm of medication errors related to the parenteral nutrition process in a large university teaching hospital. Pharmacotherapy 2009;29:966-74.

41. Seger AC, Churchill WW, Keohane CA, et al. Impact of robotic antineoplastic preparation on safety, workflow, and costs. J Oncol Pract 2012:8:344-9.

42. Skouroliakou M, Konstantinou D, Papasarantopoulos P, et al. Computer assisted total parenteral nutrition for pre-term and sick term neonates. Pharm World Sci 2005;27:305-10.

43. Wheeler DW, Degnan BA, Sehmi JS, et al. Variability in the concentrations of intravenous drug infusions prepared in a critical care unit. Intensive Care Med 2008;34:1441-7.

44. Moniz TT, Chu S, Tom C, et al. Sterile product compounding using an i.v. compounding workflow management system at a pediatric hospital. Am J Health Syst Pharm 2014;71:1311-7.

45. Nguyen HT, Pham HT, Vo DK, et al. The effect of a clinical pharmacist-led training programme on intravenous medication errors: a controlled before and after study. BMJ Qual Saf 2014;23:319-24
46. Niemann D, Bertsche A, Meyrath D, et al. A prospective three-step intervention study to prevent medication errors in drug handling in paediatric care. J Clin Nurs 2015;24:101-14.

47. Tavakoli-Ardakani M, Omidi S, Eshraghi A, et al. Medication errors in administration of chemotherapeutic agents: an observational study. Iran J Pharm Sci 2013;9:1-11.

48. Ding Q, Barker KN, Flynn EA, et al. Incidence of intravenous medication errors in a chinese hospital. Val Health Reg Issues 2015;6:33-9.

49. Khalili H, Sheikhbabayi M, Samadi N, et al. Bacterial contamination of single- and multiple-dose vials after multiple use and intravenous admixtures in three different hospitals in Iran. Iran J Pharm Res 2013;12:205-9.

50. Aruna C, Nagaraju A, Swathi K, et al. Errors in the intravenous drug administration in general medicine department of tertiary care teaching hospital. Journal of Global Trends in Pharmaceutical Sciences 2015;6:2628-34.

51. Campino A, Arranz C, Unceta M, et al. Medicine preparation errors in ten Spanish neonatal intensive care units. Eur $J$ Pediatr 2016;175:203-10.

52. Helder OK, Kornelisse RF, Reiss IK, et al. Disinfection practices in intravenous drug administration. Am J Infect Control 2016;44:721-3.

53. Rashed AN, Tomlin S, Aguado V, et al. Sources and magnitude of error in preparing morphine infusions for nurse-patient controlled analgesia in a UK paediatric hospital. Int $\mathrm{J}$ Clin Pharm 2016;38:1069-74.

54. Reece KM, Lozano MA, Roux R, et al. Implementation and evaluation of a gravimetric i.v. workflow software system in an oncology ambulatory care pharmacy. Am J Health Syst Pharm 2016;73:165-73.

55. Yin TS, Said MM, Rahman RA, et al. An investigation of errors: The preparation and administration of parenteral medications in an intensive care unit of a tertiary teaching hospital in Malaysia. Int $J$ Pharm Pharm Sci 2016:8:325-9.

56. Terkola R, Czejka M, Bérubé J. Evaluation of real-time data obtained from gravimetric preparation of antineoplastic agents shows medication errors with possible critical therapeutic impact: Results of a large-scale, multicentre, multinational, retrospective study. J Clin Pharm Ther 2017;42:446-53.

57. van den Heever A, Scribante J, Perrie H, et al. Microbial contamination and labelling of self-prepared, multi-dose phenylephrine solutions used at a teaching hospital. Southern African Journal of Anaesthesia and Analgesia 2016;22:175-9.

58. Rich DS, Fricker MP, Cohen MR, et al. Guidelines for the safe preparation of sterile compounds: results of the ISMP Sterile Preparation Compounding Safety Summit of October 2011. Hosp Pharm 2013;48:282-94. 